\title{
On the Global Distribution of Persistent Organic Pollutants
}

\author{
Pilar Fernández ${ }^{\star}$ and Joan O. Grimalt
}

\begin{abstract}
The global distribution of persistent organic pollutants (POPs) has become one of the main environmental problems in the last decade. This article gives an overview of the main contributions to the knowledge of the atmospheric transport and accumulation mechanisms of POPs in remote areas, based on their analysis in selected environmental compartments from high altitude mountain regions of Europe. The studies indicated that transport and deposition of polycyclic aromatic hydrocarbons are mainly linked to atmospheric particles. Consequently, wet and dry deposition are the main removal processes of these compounds from the atmosphere, resulting in a significant influence of regional sources. In contrast, gas exchange seems to be the main input mechanism of organochlorine compounds (OCs) from the atmosphere to terrestrial and aquatic systems. For these compounds, an altitude dependence of their accumulation in fish muscle and sediments was detected, with a major retention of the less volatile compounds (vapor pressure $\leq 10^{-2.5} \mathrm{~Pa}$ ) in the locations situated at higher altitude, those of lower annual average temperature, whereas no relationship was observed for more volatile OCs. The results represent a new aspect in the Global Distillation Effect theory as proposed for semivolatile persistent pollutants, indicating that this transport mechanism not only involves transfer from low to high latitudes, but also preferential accumulation of the less volatile compounds in high altitude regions of mid-latitude areas.
\end{abstract}

Keywords: Global distribution models · Organochlorine compounds · Persistent organic pollutants . Polycyclic aromatic hydrocarbons $\cdot$ Remote lakes

\section{Introduction}

\section{POPs}

Great concern has arisen during the last decade about the occurrence of the socalled persistent organic pollutants (POPs, Table) in the environment. Their semivolatile character and high environmental half-lives result in long-range atmospheric transport and global planetary distribution, which is especially relevant due to their potential toxicity and strong tendency to bioaccumulate in living organisms. All these factors have led to the implemen-

${ }^{*}$ Correspondence: Dr. P. Fernández Environmental Chemistry Department Institute of Chemical and Environmental Research of Barcelona Jordi Girona, 18

E-08034-Barcelona, Spain

Tel.: +34934006100

Fax: +34 932045904

E-Mail: pfrqam@iiqab.csic.es tation of international measures for the control of their release into the environment [1-3].

Most POPs are organochlorine compounds (OCs) used for diverse applications, both industrial and agricultural (Table). Their intentional use has been restricted or banned in many developed countries, but in some cases, e.g. 4,4'-DDT, their application in developing countries is allowed or unregulated. Other POPs like dioxins, furans or polycyclic aromatic hydrocarbons $(\mathrm{PAH})$ are mainly produced in combustion processes, which complicates even more the control of their emissions to the environment. The list of anthropogenic compounds to be considered as potential POPs is not closed and different candidates are being evaluated for addition to the UNECE POP Protocol [4].

\section{Global Distribution Mechanisms}

One peculiarity of the global POPs distribution is their accumulation in the environmental compartments situated at higher latitude, resulting in an enrichment of the

Table. UNEP POP list adopted in the Stockholm Convention on persistent organic pollutants [3]. The compounds specifically analyzed in this study are underlined.

$\begin{array}{ll}\text { Aldrin } & \text { Hexachlorobenzene } \\ \text { Toxaphene } & \text { Mirex } \\ \underline{\text { DDTs }} & \text { Dioxines } \\ \underline{\text { PCBs }} & \text { Furans } \\ \text { Heptachlor } & \text { Chlordane } \\ \text { Dieldrin } & \text { Chlordeconea } \\ \text { Endrin } & \text { Hexabromobiphenyl }{ }^{\text {a }} \\ \text { Polycyclic Aromatic Hydrocarbons }^{\text {a }} & \text { Hexachlorocyclohexanes }^{\text {a }} \\ { }^{\text {aChemicals included in the UN-ECE POP Protocol [1] }}\end{array}$


concentrations of some POPs in polar ecosystems to levels that sometimes exceed human consumption guidelines [5]. A global distribution model has been proposed to explain this latitudinal gradient, referred to as the Global Distillation Effect and Cold Condensation [6][7]. This model is based on physico-chemical POP properties, their sources to the environment, and the Earth climatic conditions.

The atmosphere is the main transport media for the global distribution of POPs. Once in the environment and based on their semivolatile character $\left(\mathrm{Pv}\right.$ between $10^{-4}$ and $10^{-11}$ atm at $25^{\circ} \mathrm{C}$ ), these compounds can be encountered in the gas phase, in association with atmospheric particles or distributed between these two phases [8-10] (Fig. 1). This distribution is important for their long-range transport, since preferential association with atmospheric particles increases their removal rate from the atmosphere by dry and wet deposition processes, which ultimately limits their travel distance from the sources. In addition, during atmospheric transport, POPs undergo a number of processes like degradation, deposition to soils, vegetation or water bodies, revolatization, sedimentation, and bioaccumulation (Fig. 1), which will determine their fate in the global environment. The overall significance of all these processes depends on the climatic conditions and physico-chemical properties of each compound.

As many physico-chemical properties governing the environmental fate of POPs are temperature dependent, the theory of the Global Distillation Effect predicts the transport of gas phase contaminants from the warm regions of the planet, that is tropical or temperate source areas, to colder, higher latitude regions (Fig. 2). This temperature decrease will have an effect on the vapor pressure and Henry's law constant of these compounds, increasing their tendency to condense and accumulate in surfaces like atmospheric particles, soils, vegetation, and aquatic ecosystems, from where they can enter into the food chains. Depending on their volatility, different POPs will condense at different ambient temperatures resulting in a fractionation of these chemicals in the Earth.

The transfer from low to high latitudes takes place in successive condensation/ volatization steps known as the grasshopper effect by which these compounds are exchanged between air and terrestrial surfaces according to seasonal temperature changes at mid-latitudes (Fig. 2). According to this mechanism, most POPs would be ultimately accumulated in the polar regions where the ambient temperature is low

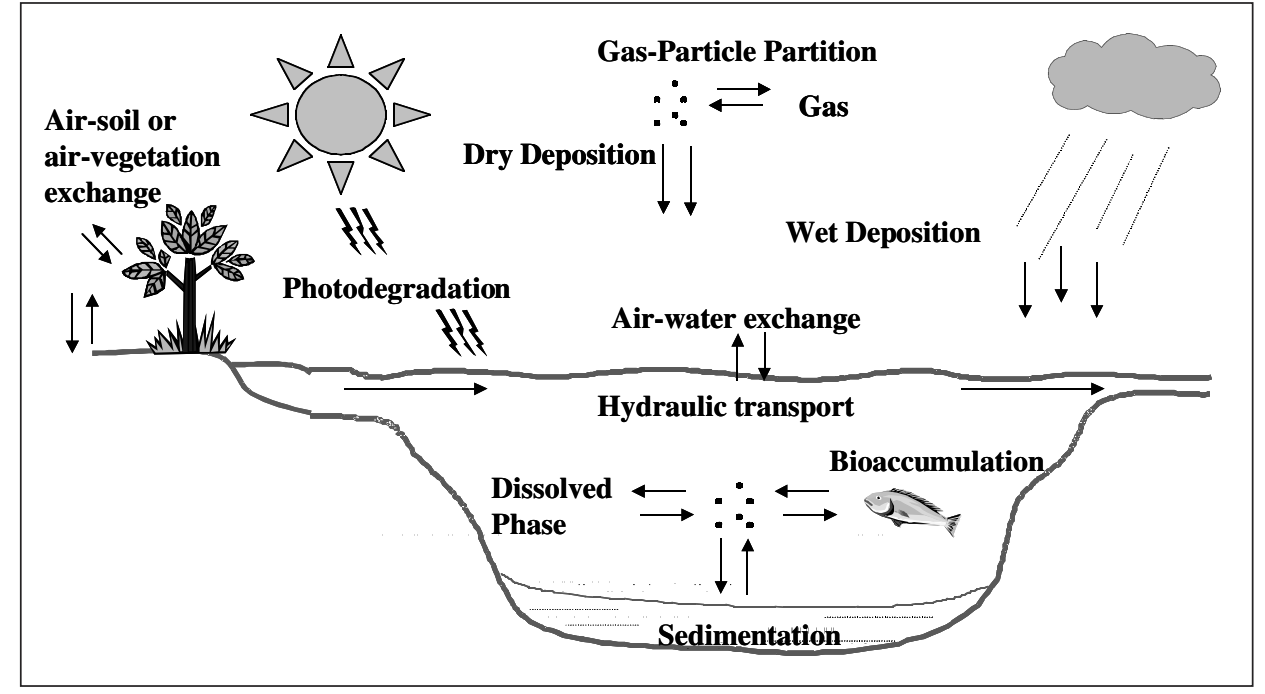

Fig. 1. Main environmental processes during long-range atmospheric transport of POPs.

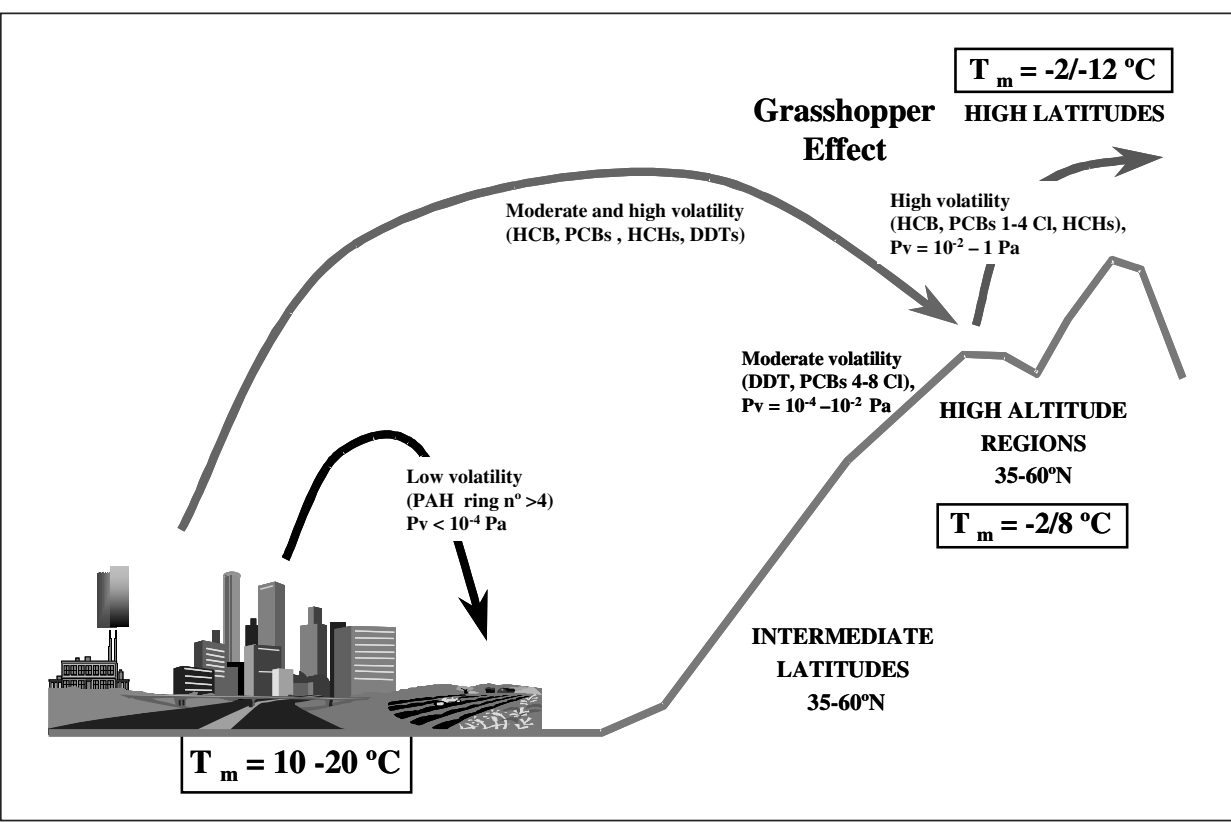

Fig. 2. Schematic representation of the Global Distillation Effect through the grasshopper mechanism in temperate areas. Pv, vapor pressure of the subcooled liquid, Tm, mean air temperature in each region.

enough to trap them, an effect termed as the cold finger or cold trap [11].

A number of studies published in the last decade reported global POP concentration patterns that are consistent with this distribution mechanism. For example, hexachlorobenzene (HCB) levels in tree bark exhibit a significant increase from the equator to northern regions [12][13]. Similar latitudinal gradients have also been described for polychlorinated biphenyls (PCBs), mainly the more volatile congeners, hexachlorocyclohexanes (HCHs), and HCB in different environmental compartments such as air [14][15], sediments [16][17], and soils [18][19]. Recently, poly- brominated diphenyl ethers (PBDE), used as flame-retardants and not included in the POP list, have been found in high concentrations in Arctic ringed seals [20].

Most of these studies have focused on the polar high latitude regions. Less attention has been paid to the global distribution of these compounds in temperate areas where the main sources are located. In order to avoid local pollution effects, the study of these areas must be focused on remote sites. High mountain regions are unique ecosystems for these purposes, and lakes located in these areas constitute stable ecosystems where the influence of chronical inputs of atmospherically transported 
POPs can be monitored. Thus, acid deposition and climatic change have been reported to have deleterious effects in some of these ecosystems [21].

On the other hand, high mountain regions also provide a temperature gradient similar to that associated with latitude. In this perspective, several research projects funded by the European Union have been focused on the study of POPs (those underlined in the Table) in high altitude European mountain lakes (Fig. 3). POPs levels in sediments, lake water, fish, soils, atmospheric deposition, snow, and air have been examined in order to obtain an integrated picture of the dynamics of these compounds in these ecosystems.

The lakes selected for study should be situated above the regional timberline, and should be devoid of human disturbance both in the lake or catchment area. In other words, changes in their hydrology and ecology should only respond to air pollution, climate and natural variability. All lakes selected for study are oligothrophic and covered with ice for a long period of the year [22]. With the exception of Lake Redó, all these lakes are small shallow-water bodies, with small catchment areas, constituted essentially by bare rock with few mosses and alpine meadows. As references, other European remote zones have been included in the study such as lake Arresjøen in the Arctic, which is not a high mountain lake, but it is situated far from any pollution source, and a high mountain area located in the subtropics, Teide Mt., Tenerife Island (Spain). This area does not contain lakes, but soils and air were analyzed for comparison.

\section{Material and Methods}

\section{Sediments}

Samples were obtained by sediment coring in the deepest point of the lake, divided into sections of $0.5 \mathrm{~cm}$ in situ, and frozen until analysis. Wet samples were extracted by sonication. Different clean-up steps by alkaline hydrolysis and column adsorption chromatography were applied until OCs and PAH fractions were obtained [23].

\section{Fish}

Samples were collected following standard test-fishing procedures and dissection as described in [24]. Muscle tissue was extracted by Soxhlet with hexane/dichloromethane. Then, a clean-up step using concentrated sulphuric acid was repeated until a colorless n-hexane layer was obtained [25]. Only OC compounds were determined in fish muscle.
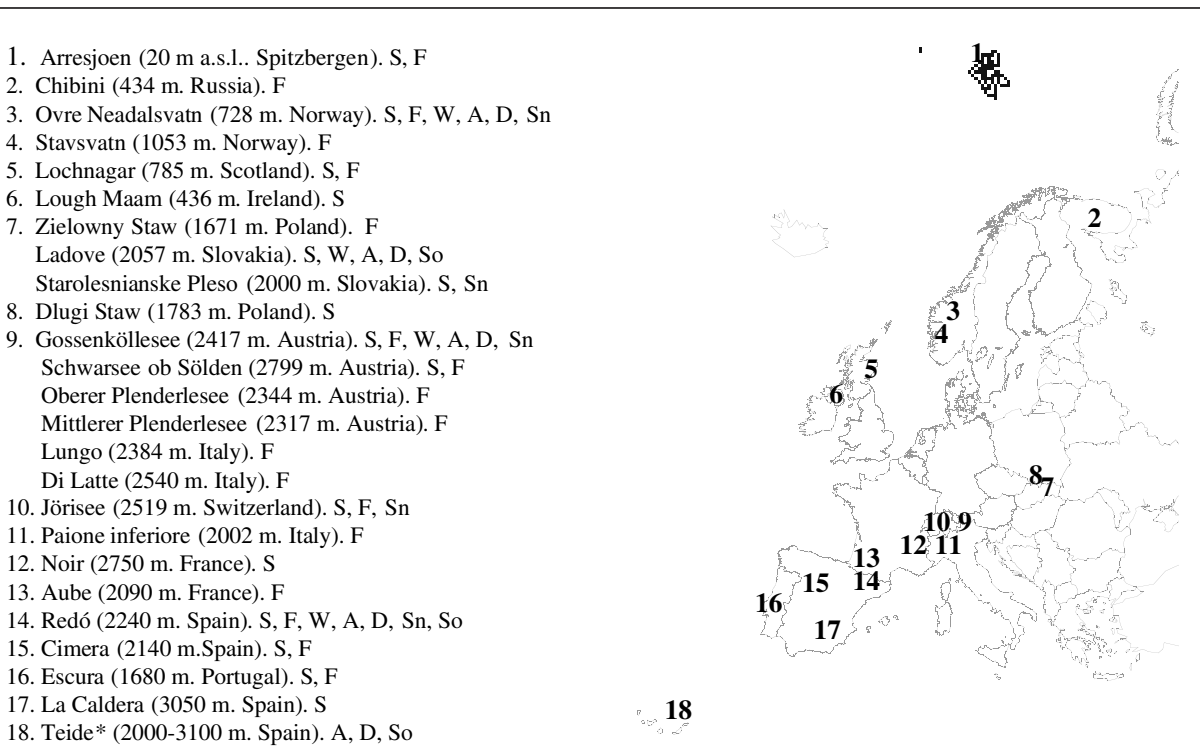

Fig. 3. High mountain lakes network with indication of their altitude above sea level and the environmental compartments analyzed in each site. S, sediments; F, fish; W, lake water; A, air; D, atmospheric deposition; Sn, snow; and So, soils. *Different altitudes in Mt. Teide (no lake present)

\section{Soils}

Soil cores were collected in an undisturbed area near the lakeshore or at different altitudes in Teide Mt. Dry samples were Soxhlet extracted as described in [26]. Three clean-up procedure steps were performed before concentration and instrumental analysis.

\section{Atmospheric Deposition and Snow}

Bulk and dry-wet only atmospheric deposition samples were continuously collected at high altitude sites for different periods, usually one year and a half. Detailed description of the sampling procedure has been reported elsewhere [27][28]. Briefly, samples were filtered on-site through preweighed glass fiber filters (Whatman, $\mathrm{GF} / \mathrm{B}, 45 \mathrm{~mm}$ diameter, $1 \mu \mathrm{m}$ pore size), and the filtrates were solid-phase extracted with C18 Empore disks (47 mm diameter, $0.5 \mathrm{~mm}$ thickness)

Snow cores were taken at the time of maximum snow accumulation, left to melt and treated as wet deposition samples.

Freeze-dried glass fiber filters were extracted by sonication with dichloromethane/methanol 2:1. Compounds adsorbed in the membrane extraction disks were eluted subsequently with methanol $(5 \mathrm{ml})$, cyclohexane $(5 \mathrm{ml})$ and dichloromethane $(5 \mathrm{ml})$. Both extracts were fractionated with aluminum oxide as described in [29].

\section{Water}

Lake water samples (about 100 l) were taken at several depths in different seasons including periods of ice-free and ice-cov- ered lake surface. The sampling device was a stand-alone pump combined with in situ filtration and solid-phase extraction (Infiltrex II, Axys Environmental Systems Ltd, Sidney, Canada). The materials retained in the filters $(\mathrm{GF} / \mathrm{B}, 1 \mu \mathrm{m}$ nominal cutoff, Whatman) and the resins (XAD-2) were operationally defined as the particulate and the dissolved phases, respectively. Analytical procedures are described in [30][31], briefly freeze-dried filter samples were extracted by sonication with dichloromethane/methanol 2:1, hydrolyzed overnight with $6 \% \mathrm{KOH}$ in methanol, and column fractionated with $2 \mathrm{~g}$ of neutral alumina. The XAD-2 adsorbent column was eluted with $200 \mathrm{ml}$ of methanol followed by $200 \mathrm{ml}$ of dichloromethane.

\section{Air}

Atmospheric samples (approx. $100 \mathrm{~m}^{3}$, gas and particulate matter) were collected using a high-volume air sampler equipped with glass fiber filters and polyurethane foam plugs (PUFs) as described elsewhere [32][33]. Glass fiber filters were cut into small pieces and extracted with dichloromethane/methanol 2:1 in an ultrasonic bath $(3 \times 25 \mathrm{ml}, 20 \mathrm{~min}$ each). PUFs were Soxhlet-extracted with $400 \mathrm{ml}$ of n-hexane for 24 h. Further clean-up by adsorption column chromatography was performed on some samples before instrumental analysis.

\section{Instrumental Analysis}

POPs levels were determined by gas chromatography with ECD (OCs) and gas chromatography coupled to mass spectrometry in electron impact and selected. 
ion monitoring modes (PAHs). Detailed description of the chromatographic and spectrometric conditions is given in [23][26][34][35]. OC identification was confirmed by gas chromatography coupled to mass spectrometry in negative chemical ionization mode using ammonia as reagent gas [36]. Optionally, this technique was used for quantification when interferences in GC-ECD do not allow OC determination.

\section{Quality Control and Assurance}

The analysis of trace pollutants in remote areas currently involves POP determination close to the instrumental detection limit. Thus, stringent precautions have to be taken in all methodological steps to avoid sample contamination and careful calibration of the instrumental technique must be performed. Specific description of the precautions taken is described in the above reported literature referring to specific environmental compartments. In general, all materials were carefully cleaned and their purity was checked by vacuum concentration of $100 \mathrm{ml}$ to $100 \mu \mathrm{l}$ for gas chromatography analysis. A series of transport, laboratory and field blanks were processed together with the samples, which usually involved $25 \%$ of the total samples analyzed. Method detection (MDL) and quantification limits (MQL) were calculated for each sample type, only compounds above MQL were taken into account in the different studies.

Recoveries and reproducibility of the analytical procedure were evaluated by addition of surrogate standards before sample extraction. Reported values were surrogate recovery and blank corrected.

\section{Results and Discussion}

\section{Global Distribution of Persistent Organic Pollutants}

The first studies on POP levels in high mountain lakes were focused on the spatial and temporal trends of these long-range atmospheric transported pollutants. Thus, sediment cores and fish from diverse lake sites were analyzed. Interestingly, all POPs investigated were found in all lakes, even in the one located in the Arctic area, and at concentration levels that, in some cases, cannot be considered low [23]. These first studies also showed a differentiation between PAHs and OCs, since their spatial distribution reflected distinct transport and accumulation mechanisms.

PAH analysis in sediments evidenced high levels in Eastern Europe and a much lesser extent in Norway, whereas the rest of the lakes showed similar low concentrations (Fig. 4). This uniformity was also reflected in the qualitative distribution of the individual compounds, which was similar in all sites independent of their location or pollution level [23].

In addition, analysis of PAHs in other environmental compartments including air, snow, water, and soils, showed the same geographic distribution detected in sediments, indicating that lake sedimentary concentrations reflect the input loads of these compounds from the atmosphere (Fig. 4). Moreover, detailed analysis on atmospheric speciation of these compounds indicated that gas phase concentrations were similar at all sites, except in Eastern Europe, whereas seasonal and the above-mentioned geographical patterns were observed for particle-associated PAH [32], pointing to a higher influence of regional sources than in the gas phase. All these results suggest that atmospheric particles are the main transport media for these compounds [32][37] and that PAH deposition and accumulation in
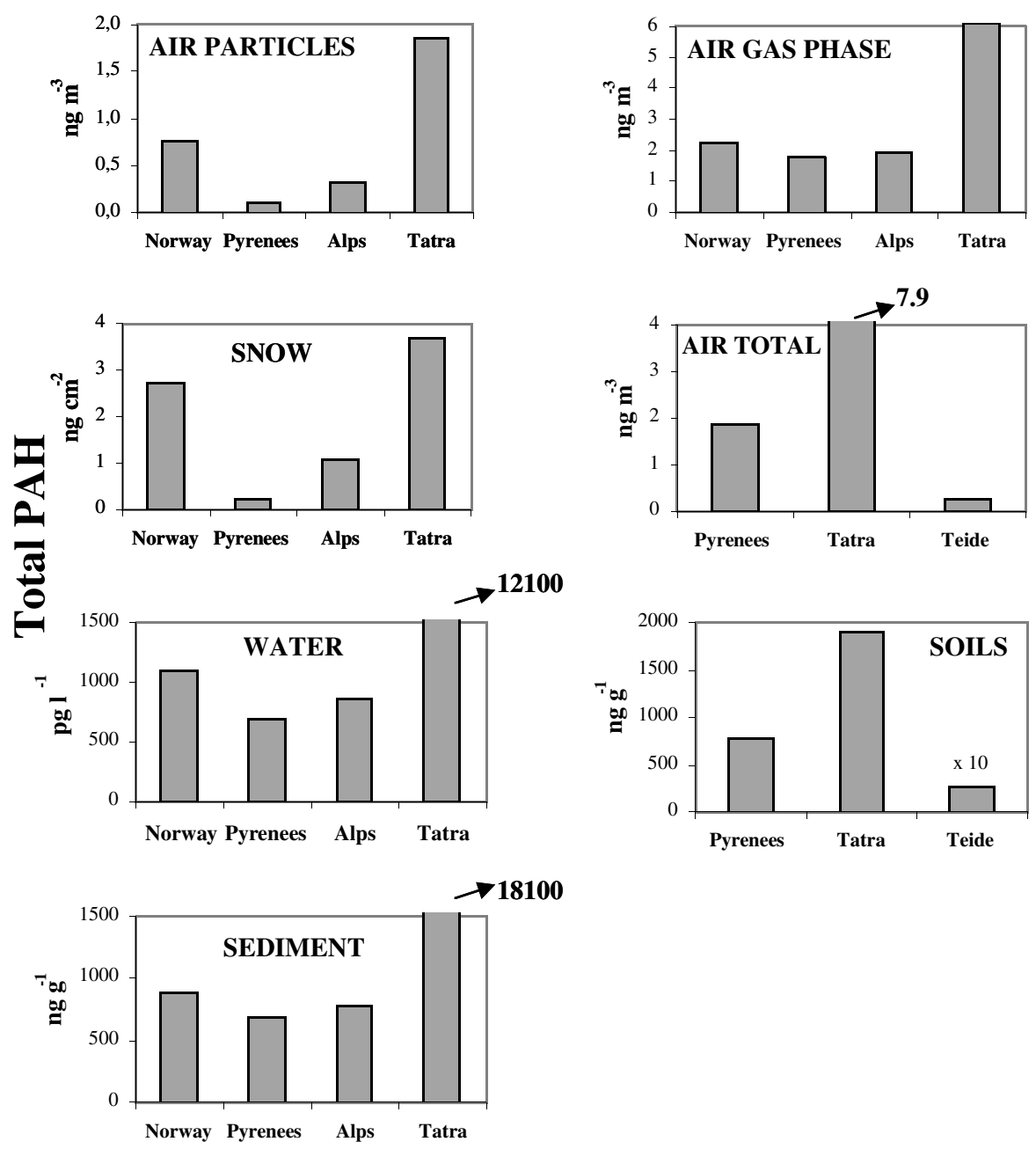

Fig. 4. Total PAH levels (sum of 23 parent compounds from fluorene to coronene) in different environmental compartments from high altitude regions. Site identification based on Fig. 3 numbers: Norway 3, Pyrenees 14, Alps 9, Tatra 7, and Teide 18. remote lakes is related directly to particle deposition through both dry and wet processes. Thus, PAHs should travel over moderate distances and, therefore, their composition in remote sites should reflect influences from regional sources.

In contrast, no common geographical trend was observed for the concentration of OCs in sediments and fish of high altitude mountain lakes, although the highest sedimentary levels also occur in the Tatra Mts (Fig. 5). The different behavior of PAHs and OCs is evidenced further when considering the environmental compartments mentioned above, since they do not show ence for their accumulation in fish muscle and sediments (expressed as inventories) was observed, in that the less volatile compounds (vapor pressure $\leq 10^{-2.5} \mathrm{~Pa}$ ) were retained to a major extent in the locations at altitude, those of lower annual average temperature. In contrast, no altitudinal dependence was observed for the more y parallel trend for each specific location. 


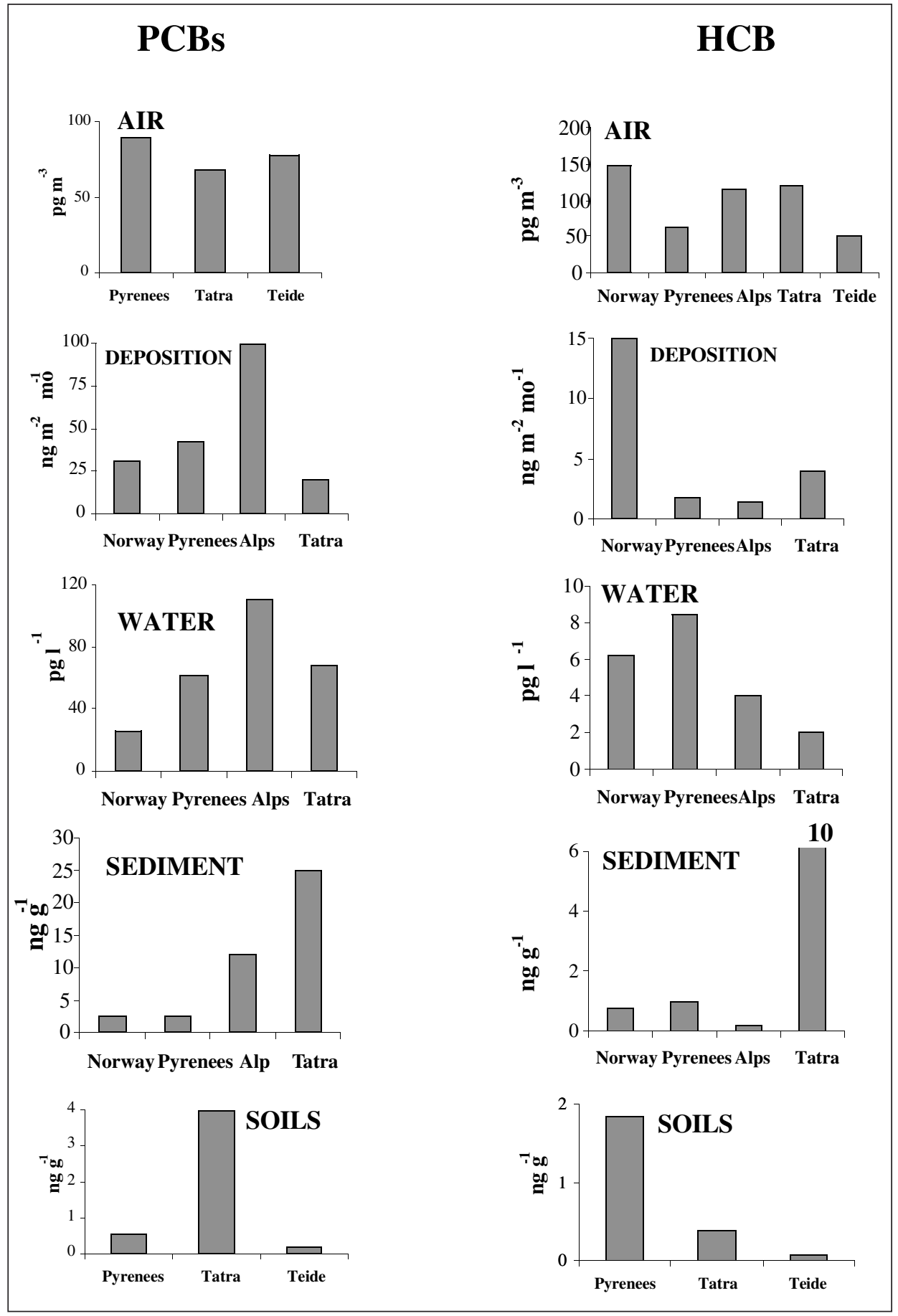

volatile OCs (Fig. 6) [35]. This trend is observed better for PCBs, since they include different congeners with a broad volatility range. Accordingly, less volatile congeners (PCB \#153, \#138 and \#180; Fig. 6) showed altitudinal correlations (in fact, temperature), whereas the more volatile congener PCB 28 did not. These different behaviors suggest the occurrence of a condensation mechanism similar to that described in the polar regions that drives the accumulation of these compounds in high altitude areas.
Fig. 5. HCB and PCBs (sum of congeners 28, $52,101,118,153,138$, and 180) in different environmental compartments from high altitude regions. Site identification as in Fig. 4.

deposition may be subsequently transferred to the atmosphere in the warm period (grasshopper effect, Fig. 2). Only a fraction of the less volatile compounds will be retained in the lake in a proportion that is defined by the local temperature.

Further evidence of this accumulation mechanism can be obtained from the qualitative PCB distribution in atmospheric deposition, water and sediments from selected sites representing different ambient temperatures [29] (Fig. 8). PCB distributions in deposition samples are very similar between sites dominated by the less chlorinated, more volatile congeners. The resulting compositions indicate a rather uniform PCB deposition load to remote high mountain lakes that is independent of local conditions. In contrast, large differences between sites are observed in lake water, sediment and/or fish (not shown), which are enriched in less volatile congeners. This enrichment is more pronounced for the sites with the lowest average annual temperature. 


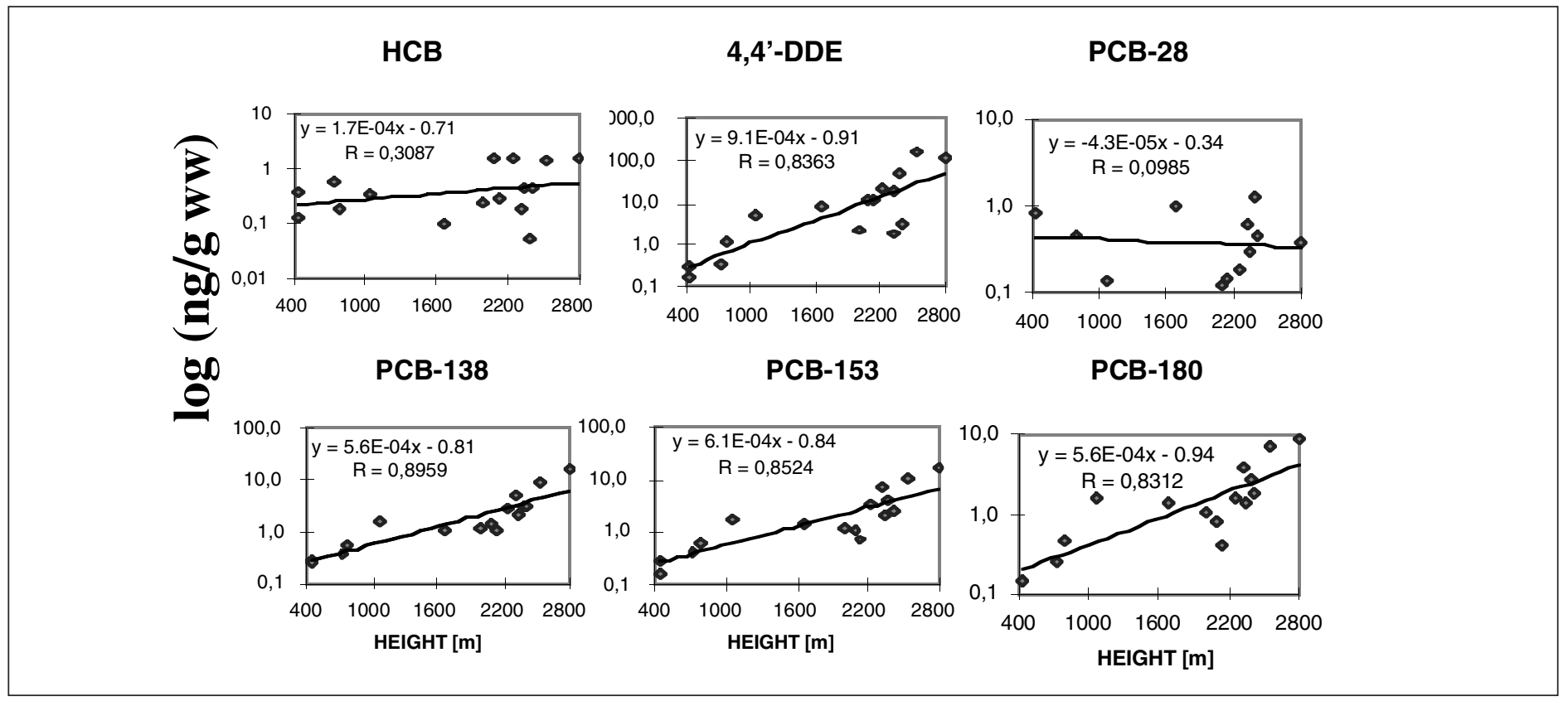

Fig. 6. Linear regression between fish content and lake altitude for selected OCs.

\section{The High Mountain Ecosystems in the Context of the Global POP Distribution}

Studies in the Canadian Rocky Mountains [39][40] also reported an altitudinal gradient of $\mathrm{OC}$ concentration measured in snow and conifer needles. In both cases, a linear correlation of the more volatile congeners with altitude was observed, whereas less volatile OCs were either unrelated or inversely correlated. The differences in altitude dependence observed between the Canadian and European high mountain regions can be explained in a first approach by differences in air temperature ranges. Samples of snow from the Canadian Rocky Mountains correspond to January temperatures between $-8{ }^{\circ} \mathrm{C}$ and $-18{ }^{\circ} \mathrm{C}$ (air temperatures are not reported in the paper about POPs levels in vegetation), while January temperatures at the European sites included in this study range between -3 and $-8^{\circ} \mathrm{C}$ for the highest lake. Thus, both observations are consistent with the Global Distillation Model proposed for POPs. As shown in Fig. 2 , more volatile compounds such as HCB or less chlorinated PCBs accumulate preferentially in areas with mean temperatures in the range of -7 and $-12{ }^{\circ} \mathrm{C}$, typically found in latitudes higher than $60^{\circ} \mathrm{N}$ or mountain areas such as those of Canada, whereas less volatile compounds condense at mean temperatures between $-3{ }^{\circ} \mathrm{C}$ and $5{ }^{\circ} \mathrm{C}$, as measured in the European lakes. However, these less volatile compounds are mobilized at mean temperatures of $5-10{ }^{\circ} \mathrm{C}$ or higher, detected in low altitude sites of temperate and tropical latitudes.
As mentioned above, the composition of snow in the European sites reflects a temperature dependence of the less and the more volatile PCBs. Thus, in addition to temperature effects, a matrix effect may also partially account for the differences between Europe and Canada. Thus, the European samples reflect that all PCBs are retained in proportion to the reciprocal of the temperature in winter, e.g. snow, but only the less volatile congeners are retained at summer temperatures, e.g. snow melting. This interpretation is consistent with the differences in PCB composition between atmospheric deposition, water and lake sediment (Fig. 8).

\section{Concluding Remarks}

PAHs are mainly transported in association with particles and their distribution in remote areas, including high mountain regions essentially reflects regional contributions. In contrast, OC concentrations in high altitude sites do not reflect nearby sources but are distributed as predicted by the global distillation model. That is, according to their physico-chemical properties and air temperatures. Thus, high altitude ecosystems accumulate POPs to the same extent as high latitude regions, which receive contaminants by long-range atmospheric transport. As a consequence, there is a net transfer of toxic and persistent chemicals from mid-latitude source areas to high altitude or latitude remote regions. Other factors can also influence and enhance POP accumulation in cold regions, such as reduced degradation rates due to lower temperatures or the presence of snow and ice for long periods. In this respect, there is a lack of knowledge in the partitioning behavior of organic chemicals between air and snow and ice, and how these phases alter the air exchange processes between these pollutants and the aquatic and terrestrial surfaces.

The understanding of the global distribution mechanisms of persistent organic pollutants is a key issue for the assessment of their true impact in the environment. Transport and fate of POPs in the environment result from a complex balance between inputs and losses, in which the interaction between all compartments, biotic and abiotic, has to be considered. Thus, the significant role of oceanic biogeochemical processes in the global dynamics and fate of POPs, specifically phytoplankton uptake and subsequent sink to deep waters, has been recently stated [41], but even in this context, POP distribution is mainly governed by their physico-chemical properties and temperature.

\section{Acknowledgements}

Many people have contributed either directly or indirectly to this study. In particular, we would like to thank R.M. Vilanova, L. Berdié, G. Carrera, B. van Drooge, and S. Ribes for the analysis of most of the samples described in this work. We are also grateful to H. Thies, U. Nickus, and R. Psenner (Innsbruck University, Austria), M. Ventura, L. Camarero, and J. Catalán (CEAM-CSIC, Spain), B. Rosseland and L. Lien (NIVA, Norway), E. Stuchlik (Uni- 
A

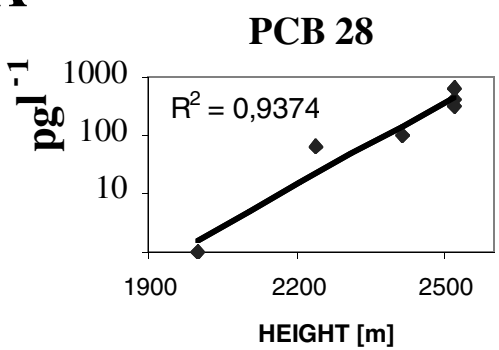

PCB 118

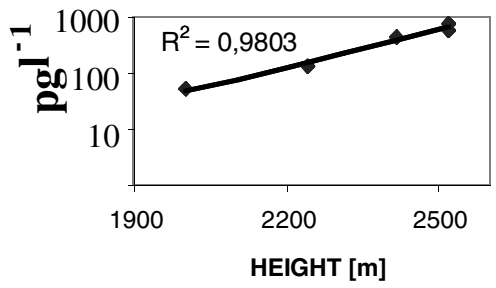

B

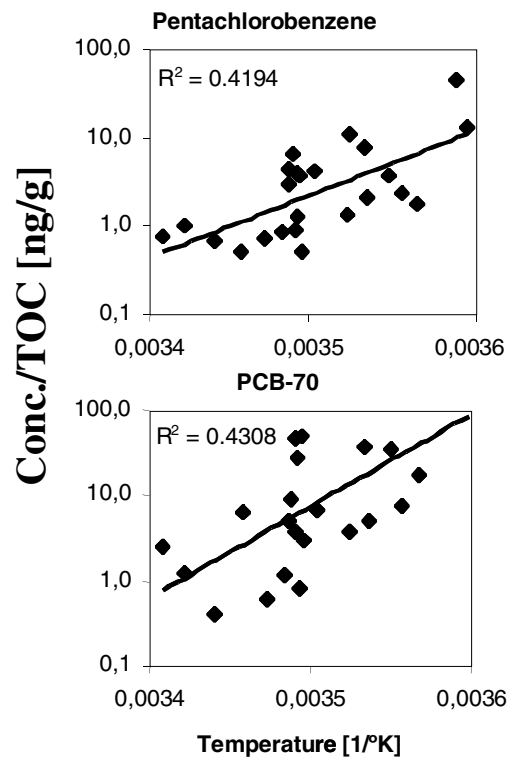

PCB 52

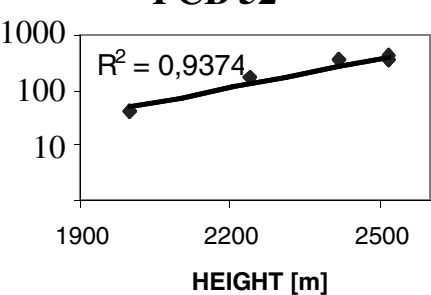

PCB 153

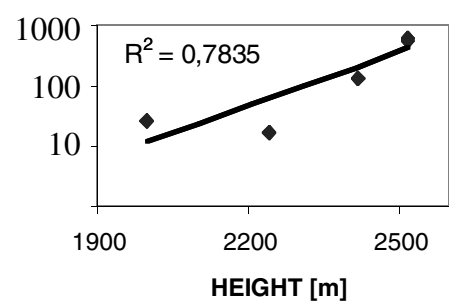

HEIGHT [m]
PCB 101

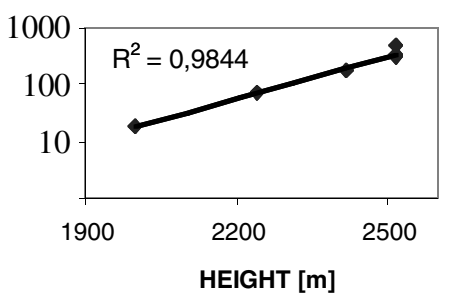

PCB 138

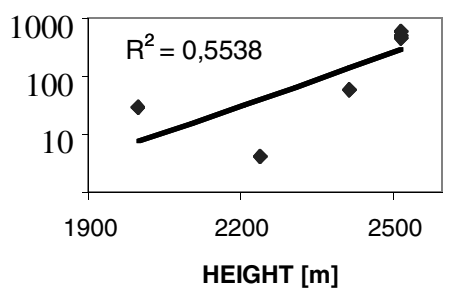

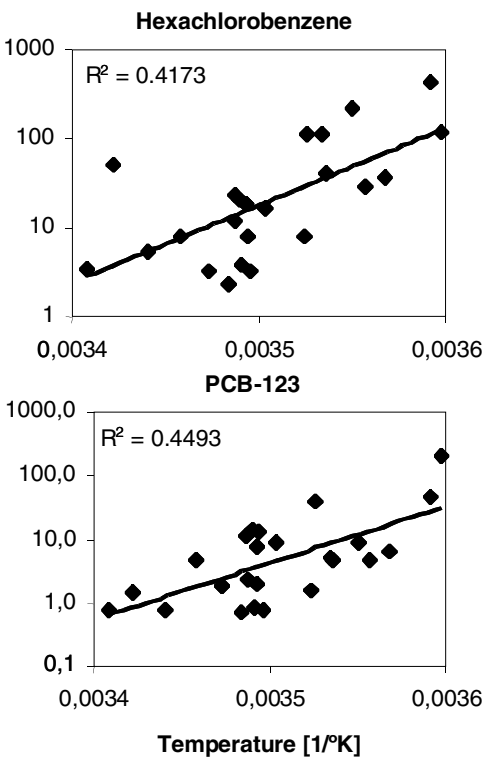

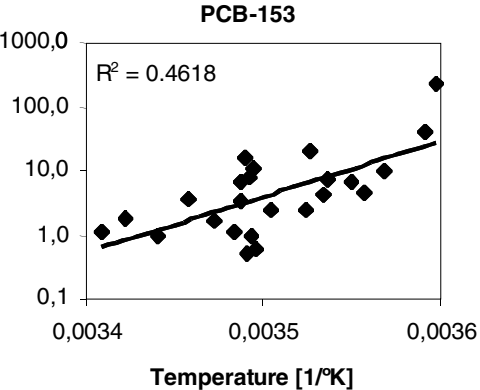

Fig. 7. Linear relation of (A) PCBs congeners in snow versus lake altitude, and (B) OCs in soils versus mean air temperature for high altitude sites. Concentration in soils related to organic carbon content (TOC). 


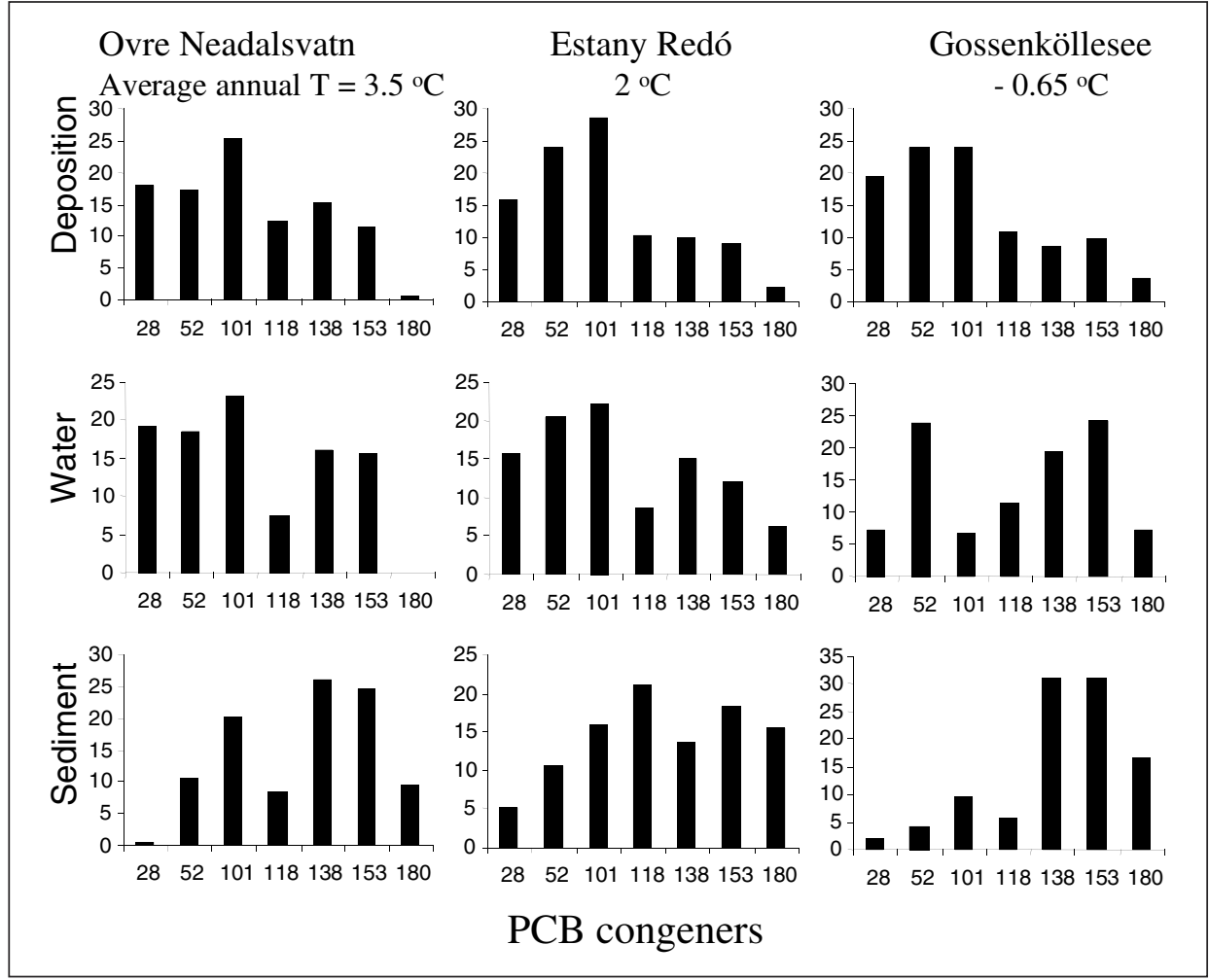

Fig. 8. Qualitative distribution of PCB congeners in selected environmental compartments of European high mountain lakes.

versity of Prague, Czech Republic), and S.T. Patrick (University College London, UK) for sediment and atmospheric deposition sampling, and useful cooperation in the air and lake water sampling campaigns. We thank $\mathrm{C}$. Torres and $\mathrm{E}$. Cuevas (National Institute of Meteorology, Spain) for soil sampling on Teide Mt. Technical assistance in GC and GC-MS instrumental analysis from R. Mas, R. Chaler, and D. Fanjul is also acknowledged.

Financial support for this study was provided by EU Environmental projects AL:PE II, MOLAR, EMERGE, and GLOBAL-SOC.

Received: July 5, 2003

[1] UN-ECE 'Protocol to the 1979 convention on long range transboundary air pollution on persistent organic pollutants and executive body decision 1998/2 on information to be submitted and the procedure for adding substances to annexes I, II or III to the protocol on persistent organic pollutants. ECE/EB.AIR/60', United Nations, New York and Geneva, 1998.

[2] N. Eckely, 'From regional to global assessment: learning from persistent organic pollutants. ENRP Discussion Paper 200023', Kennedy School of Government, Harvard University, 2000.

[3] UNEP, Stockholm convention on persistent organic pollutants. Document : UNEP/POPS/CONF/4. Available from: http://irptc.unep.ch/pops2001, 2001.

[4] D. Lerche, E.v.d. Plassche, A. Schwegler, F. Balk, Chemosphere 2002, 47, 617.
[5] AMAP 'Artic Pollution Issues: A State of the Arctic Environment Report', Arctic Monitoring and Assessment Programme, Oslo, 1998, pp 188.

[6] F. Wania, D. Mackay, Ambio 1993, 22, 10.

[7] F. Wania, D. Mackay, Science Total Environ. 1995, 160/161, 211.

[8] M. Aceves, J.O. Grimalt, Environ. Sci. Technol. 1993, 27, 2896.

[9] J.C. Duinker, F. Bouchertall, Environ. Sci. Technol. 1989, 23, 57.

[10] H. Kaupp, M.S. McLachlan, Atmospheric Environ. 1999, 33, 85.

[11] F. Wania, D. Mackay, Environ. Sci. Technol. 1996, 30, 390A.

[12] S.L. Simonich, R.A. Hites, Science 1995, 269, 1851.

[13] D. Calamari, E. Bacci, S. Focardi, C. Gaggi, M. Morosini, M. Vighi, Environ. Sci. Technol. 1991, 25, 1489.

[14] C. Agrell, L. Okla, P. Larsson, C. Backe, F. Wania, Environ. Sci. Technol. 1999, 33, 1149.

[15] S.N. Meijer, W.A. Ockenden, E. Steinnes, B.P. Corrigan, K.C. Jones, Environ. Sci. Technol. 2003, 37, 454.

[16] D.C.G. Muir, A. Omelchenko, N.P. Grift, D.A. Savoie, W.L. Lockhart, P. Wilkinson, G.J. Brunskill, Environ. Sci. Technol. 1996, 30, 3609.

[17] H. Iwata, S. Tanabe, N. Sakai, A. Nishimura, R. Tatsukawa, Environ. Poll. 1994, 85, 15.

[18] S.N. Meijer, E. Steinnes, W.A. Ockenden, K.C. Jones, Environ. Sci. Technol. 2002, 36,2146 .
[19] S.N. Meijer, W.A. Ockenden, A. Sweetman, K. Breivik, J.O. Grimalt, K.C. Jones, Environ. Sci. Technol. 2003, 37, 667.

[20] M.G. Ikonomou, S. Rayner, R.F. Addison, Environ. Sci. Technol. 2002, 36, 1886.

[21] S. Sommaruga-Wögrath, K.A. Koining, R. Schmidt, R. Sommaruga, R. Tessadri, R. Psenner, Nature 1997, 387, 64.

[22] AL:PE I 'Acidification of Mountain Lakes: Palaeolimnology and Ecology, AL:PE. Part 2 - Remote Mountain Lakes as Indicators of Air Pollution and Climate Change', Norwegian Institute for Water Research, 1997, pp 42.

[23] P. Fernández, R.M. Vilanova, J.O. Grimalt, Environ. Sci. Technol. 1999, 33, 3716.

[24] EMERGE, web page http://www.mountain-lakes.org.

[25] L. Berdié, J.O. Grimalt, J. Chromatogr. 1998, 823, 373

[26] A. Ribes, J.O. Grimalt, C.J.T. García, E. Cuevas, Environ. Sci. Technol. 2002, 36, 1879.

[27] G. Carrera, P. Fernández, R. Vilanova, J.O Grimalt, J. Chromatogr. 1998, 823, 189.

[28] G. Carrera, P. Fernández, R.M. Vilanova, J.O. Grimalt, Atmospheric Environ. 2001, $35,245$.

[29] G. Carrera, P. Fernández, J.O. Grimalt, M. Ventura, L. Camarero, J. Catalán, U. Nickus, H. Thies, R. Psenner, Environ. Sci. Technol. 2002, 36, 2587.

[30] R.M. Vilanova, P. Fernández, C. Martínez, J.O. Grimalt, J. Environ. Quality 2001, 30, 1286.

[31] R.M. Vilanova, P. Fernández, C. Martínez, J.O. Grimalt, Wat. Res. 2001, 35, 3916.

[32] P. Fernández, J.O. Grimalt, R.M. Vilanova, Environ. Sci. Technol. 2002, 36, 1162.

[33] B.L. van Drooge, J.O. Grimalt, C. TorresGarcía, E. Cuevas, Environ. Sci. Technol. 2002, 36, 1155

[34] A. Ribes, J.O. Grimalt, P. Fernández, Hydrobiologia 2003, submitted.

[35] J.O. Grimalt, P. Fernández, L. Berdié, R.M. Vilanova, J. Catalan, R. Psenner, R. Hofer, P.G. Appleby, L. Lien, B.O. Rosseland, J.-C. Massabuau, R.W. Battarbee, Environ. Sci. Technol. 2001, 35, 2690

[36] R. Chaler, R. Vilanova, M. Santiago-Silva, P. Fernández, J.O. Grimalt, J. Chromatogr. 1998, 823, 73.

[37] P. Fernández, G. Carrera, J.O.Grimalt, M Ventura, L. Camarero, J. Catalán, U. Nickus, H. Thies, R. Psenner, Environ. Sci. Technol. 2003, 37, 3261.

[38] J.O. Grimalt, P. Fernández, R.M. Vilanova, TheScientificWorld (www.thescientificworld.com) 2001, 1, 609.

[39] D.A. Davidson, A.C. Wilkinson, J.M. Blais, L.E. Kimpe, K.M. McDonald, D.W. Schindler, Environ. Sci. Technol. 2003, 37, 209.

[40] J.M. Blais, D.W. Schindler, D.C.G. Muir, L.E. Kimpe, D.B. Donald, B. Rosenberg, Nature 1998, 395, 585

[41] J. Dachs, R. Lohmann, W.A. Ockenden, L. Méjanelle, S.J. Eisenreich, K.C. Jones, Environ. Sci. Technol. 2002, 36, 4229. 